Supporting Information

\title{
Nonlinear Rheology of Telechelic Ionomers based on Sodium Sulfonate and \\ Carboxylate
}

Shuang Liu, ${ }^{1,2}$ Zhijie Zhang ${ }^{1}$ and Quan Chen* ${ }^{1,2}$

1. State Key Laboratory of Polymer Physics and Chemistry, Changchun Institute of Applied Chemistry, Chinese Academy of Sciences, 130022 Changchun, P. R. China

2. University of Science and Technology of China, 230026 Hefei, P. R. China

Yumi Matsumiya $^{3}$ and Hiroshi Watanabe ${ }^{3}$

3. Institute for Chemical Research, Kyoto University, Uji 611-0011, Japan

*Email: qchen@,ciac.ac.cn

Supporting Information Content:

S1 Preparation of Samples

S2 NMR and GPC Characterization

S3 DSC traces

S4 Shift factors

S5 X-ray Scattering and Transient Network Structure

S6 Nonlinear Rheological data of ionomers with $M_{\mathrm{n}}=5 \mathrm{~K}$ and $16 \mathrm{~K}$

S7 Invariants of stress and strain tensors

S8 Viscoelastic Behavior of Acidic Prepolymers 


\section{S1 Sample Synthesis}

\section{S1.1 Synthesis of 2-(1-Carboxy-1-methylethylsulfanylthiocarbonylsulfanyl)-2-methylpropionic acid used as a chain transfer agent (CTA) ${ }^{1}$}

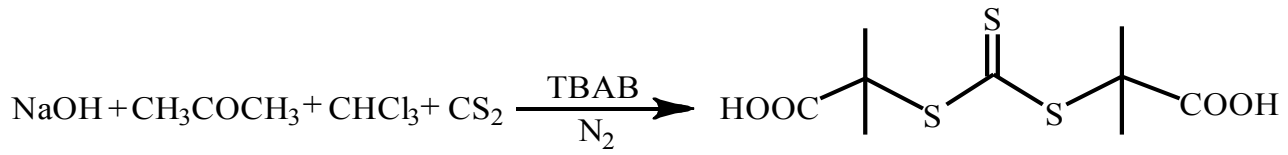

Scheme S1. Synthesis route of chain transfer agent (CTA)

Carbon disulfide $(2.74 \mathrm{~g}, 0.036 \mathrm{~mol})$, chloroform (10.75, $0.09 \mathrm{~mol})$, acetone (5.23 g, $0.09 \mathrm{~mol})$, and Tetrabutylammonium bromide (TBAB) $(0.229 \mathrm{~g}, 0.71 \mathrm{mmol})$ were mixed with $20 \mathrm{~mL}$ of mineral spirits in a $500 \mathrm{ml}$ three-necked flask with $0^{\circ} \mathrm{C}$ ice-water bath under nitrogen. Sodium hydroxide (50\%) (20.16 g, $0.252 \mathrm{~mol}$ ) was added dropwise over $90 \mathrm{~min}$ in order to keep the temperature below $10{ }^{\circ} \mathrm{C}$. The reaction mixture was stirred overnight. $100 \mathrm{~mL}$ of deionized water was then added to dissolve the solid, followed by $1 \mathrm{M}$ concentrated $\mathrm{HCl}$ to acidify the aqueous layer $(\mathrm{PH}<4)$. Stir for $30 \mathrm{~min}$ with nitrogen purge. Filter and wash the yellow solid thoroughly with lots of deionized water. It can be further purified by stirring in ethyl alcohol or by recrystallizations from ethyl alcohol to afford a yellow crystalline solid. The chemical structure was confirmed with the ${ }^{1} \mathrm{H}-\mathrm{NMR}$ as shown in Figure S1.

\section{S1.2 Synthesis of Didodecyl-1,2-phenylene-bis(methylene)bistrithiocarbonate used as a chain transfer agent (CTA) ${ }^{2}$}

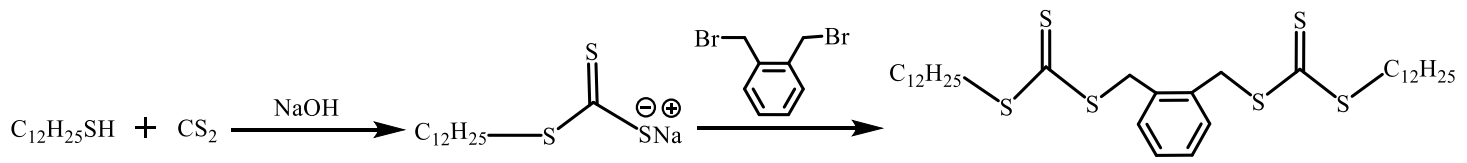

Scheme S2. Synthesis route of Didodecyl-1,2-phenylene-bis(methylene)bistrithiocarbonate RAFT agent

The bis-trithiocarbonate RAFT agent (bis-RAFT) was synthesized via a two-step phase transfer catalyzed reaction, as shown in Scheme S2. 1-dodecanthiol $(9.05 \mathrm{~g}, 0.045 \mathrm{~mol})$ and Aliquat $336(0.72 \mathrm{~g}$, $0.0018 \mathrm{~mol}$ ) were mixed with $60 \mathrm{~mL}$ toluene into a $500 \mathrm{ml}$ three-necked flask with $0^{\circ} \mathrm{C}$ ice-water bath under nitrogen. Sodium hydroxide (50\%) $(3.63 \mathrm{~g}, 0.046 \mathrm{~mol})$ was injected into the flask. After $15 \mathrm{~min}$, carbon disulfide dissolved in $20 \mathrm{~mL}$ toluene $(3.44 \mathrm{~g}, 0.045 \mathrm{~mol})$ was injected into the solution. After stirring of $15 \mathrm{~min}$, o-xylylene dibromide dissolved in $30 \mathrm{~mL}$ toluene $(5.94 \mathrm{~g}, 0.023 \mathrm{~mol})$ was added to the reactor and the mixed solution was stirred at room temperature under nitrogen for $12 \mathrm{~h}$, and terminated by adding $100 \mathrm{~mL}$ deionized water and stirring for $30 \mathrm{~min}$. The mixture was poured into a separation funnel, and a yellow toluene layer was collected and washed with deionized water 3 times. The product (yellow solid) was recovered by rotational evaporation, and further purified by 
recrystallization in hexane (yellow powder). The chemical structure was confirmed with the ${ }^{1} \mathrm{H}-\mathrm{NMR}$ as shown in Figure S2.

\section{S2 NMR and GPC Characterization}

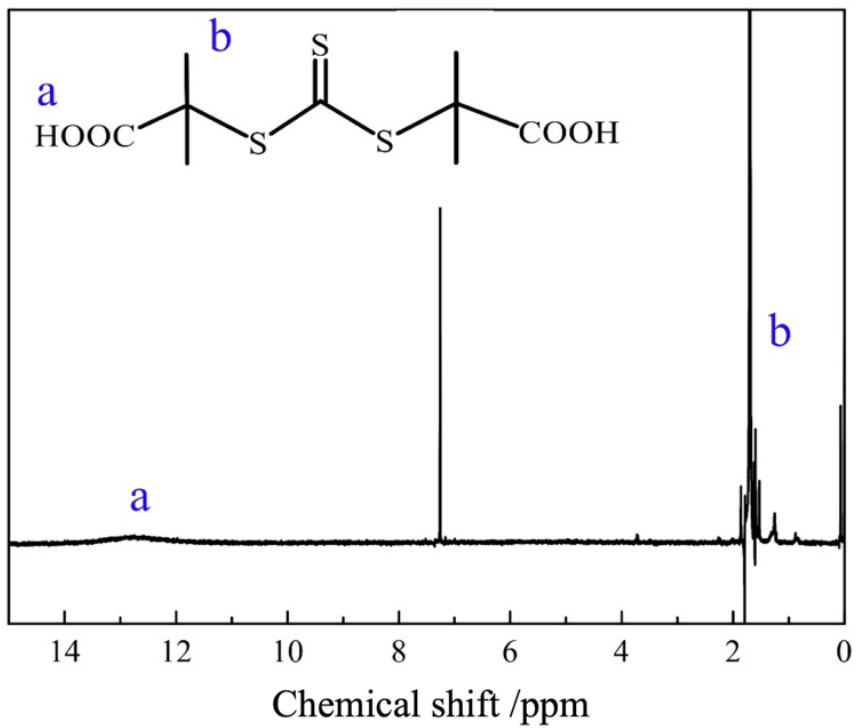

Figure S1. ${ }^{1} \mathrm{H}$ NMR spectra of carboxylated functionalized chain transfer agent.

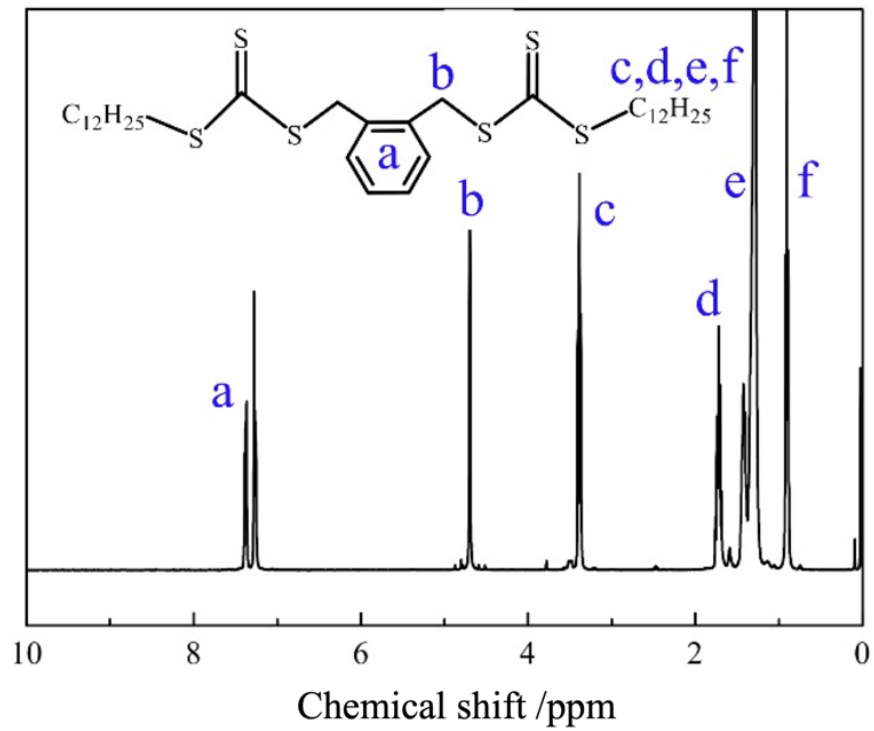

Figure S2. ${ }^{1} \mathrm{H}$ NMR spectra of bis-trithiocarbonate RAFT agent (bis-RAFT). 


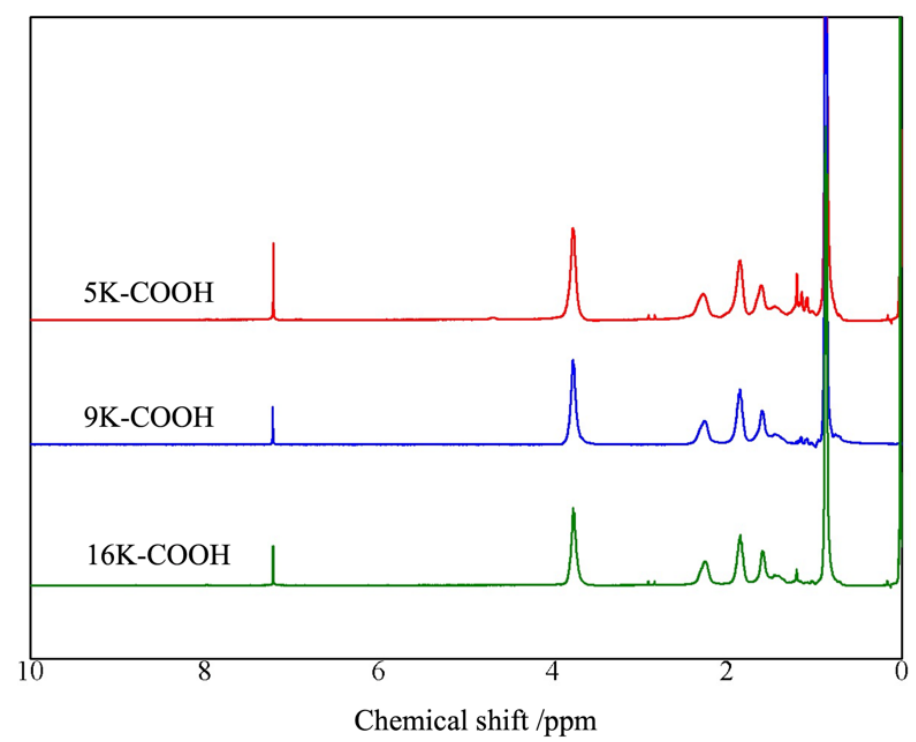

Figure S3. ${ }^{1} \mathrm{H}$ NMR spectra of telechelic carboxylated prepolymers.

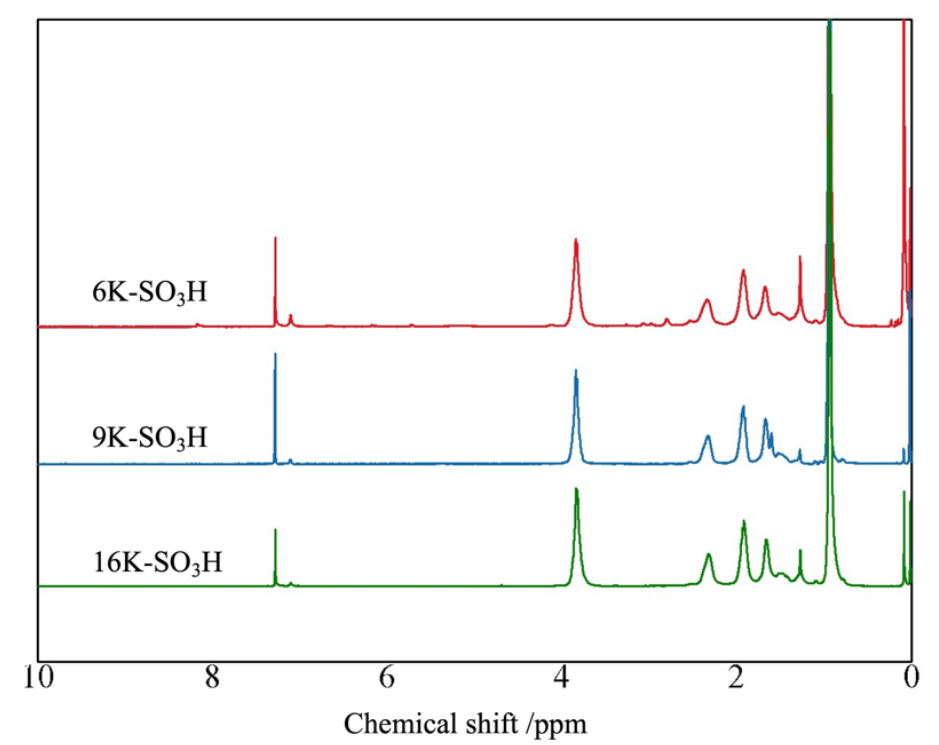

Figure S4. ${ }^{1} \mathrm{H}$ NMR spectra of Telechelic sulfonated sample $\mathrm{HO}_{3} \mathrm{~S}-\mathrm{PiBA}-\mathrm{SO}_{3} \mathrm{H}$.
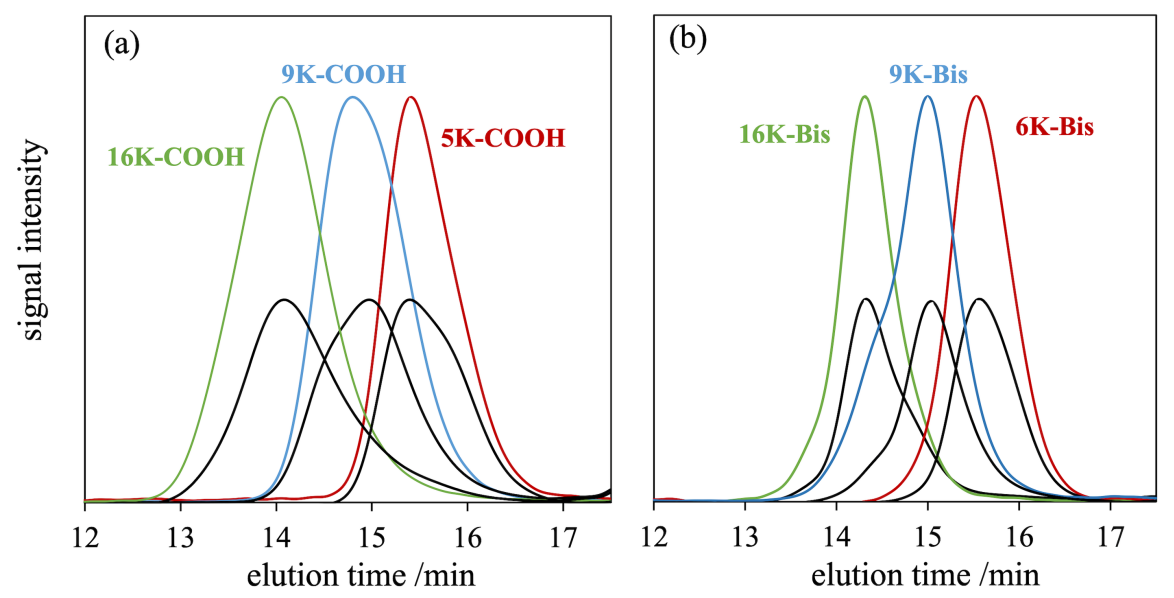

Figure S5. GPC analysis of telechelic carboxylated (a) and bis prepolymers (b). The colored and black curves show signals from the low-angle light scattering (LS) and refractive index (RI) detectors, respectively. The elution solvent is THF. 


\section{S3 DSC traces}

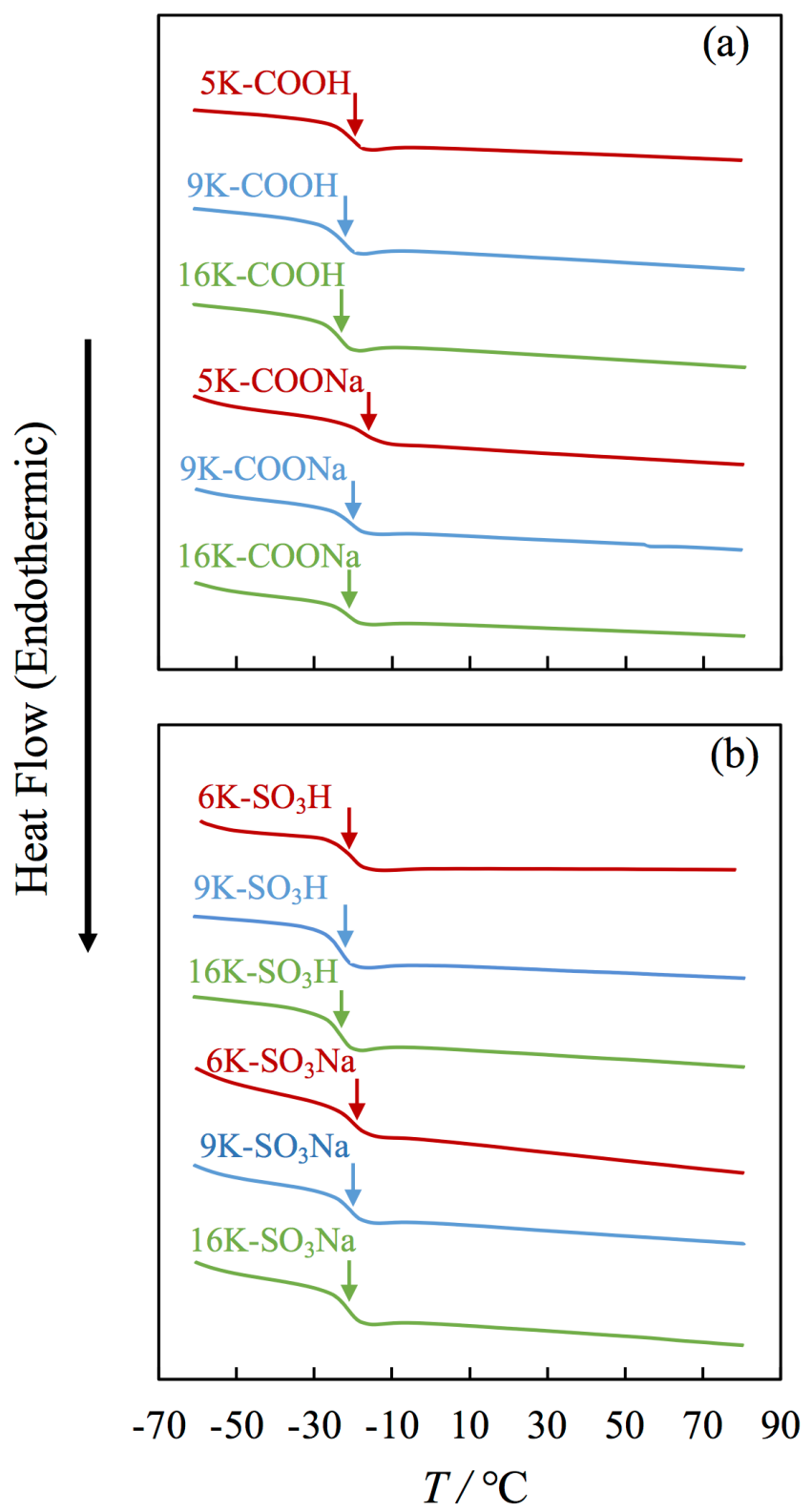

Figure S6. DSC data of (a) $M_{\mathrm{n}}-\mathrm{COOH}$ and $M_{\mathrm{n}}-\mathrm{COONa}$, and (b) $M_{\mathrm{n}}-\mathrm{SO}_{3} \mathrm{H}$ and $M_{\mathrm{n}}-\mathrm{SO}_{3} \mathrm{Na}$. The arrows show $T_{\mathrm{g}}$. 


\section{S4 Shift factors}

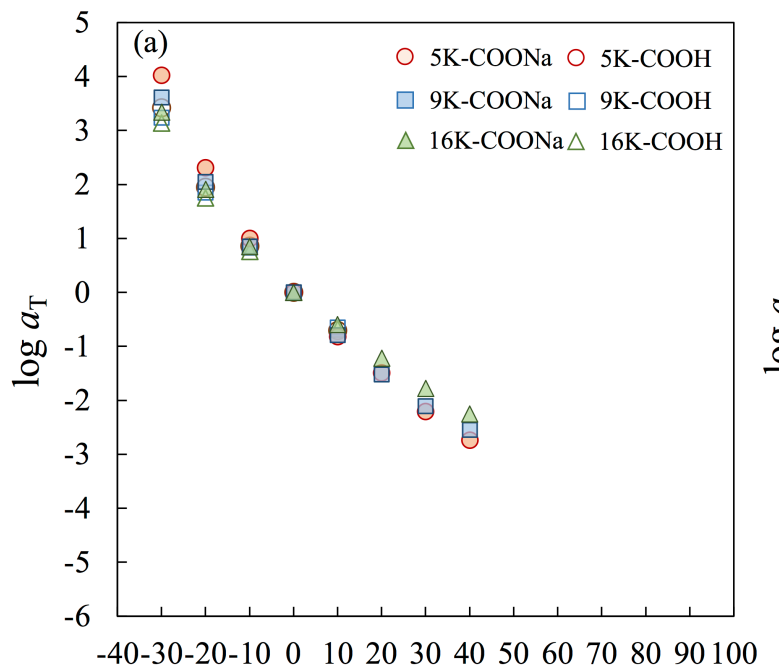

$T-T_{\mathrm{r}} /{ }^{\circ} \mathrm{C}$

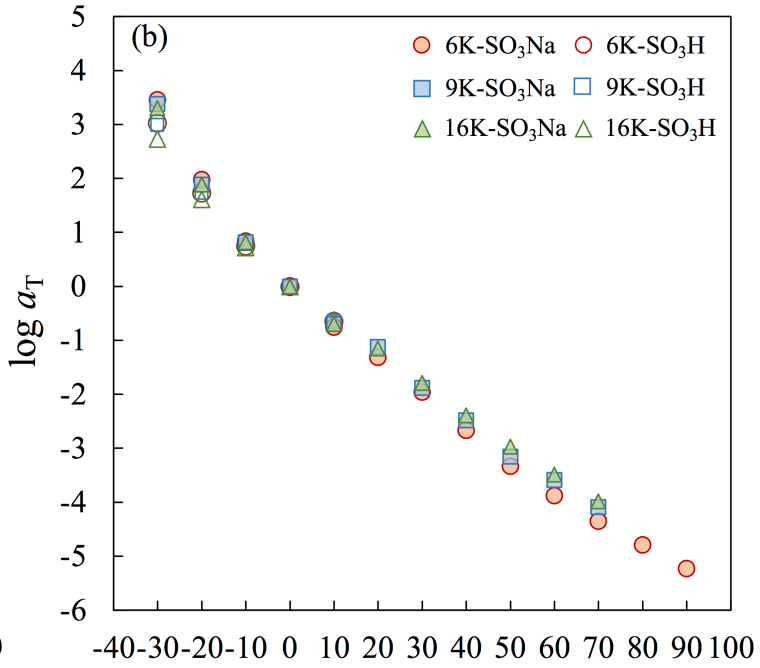

$T-T_{\mathrm{r}} /{ }^{\circ} \mathrm{C}$

Figure S7. tTs shift factors $a_{\mathrm{T}}$ of all acid prepolymers and ionomers samples for (a) $M_{\mathrm{n}}-\mathrm{COOH}$ and $M_{\mathrm{n}}-\mathrm{COONa}$, and (b) $M_{\mathrm{n}}-\mathrm{SO}_{3} \mathrm{H}$ and $M_{\mathrm{n}}-\mathrm{SO}_{3} \mathrm{Na} . \quad a_{\mathrm{T}}$ is plotted against $T-T_{\mathrm{r}}$

\section{S5 X-ray Scattering and Transient Network Structure}

\section{S5.1 Lorentzian functions fitting of X-ray scattering data.}

X-ray scattering profiles of all ionomer samples show three scattering peaks, reflecting backbonebackbone correlation, amorphous scattering of the side chains, inter-aggregate interference, and a power law-like behavior following at very low $q$; cf. Figure 3 in the main text. We use three Lorentzian functions and one power-law term to fit the scattering profile,

$$
I(q)=L_{\mathrm{a}}(q)+L_{\mathrm{b}}(q)+L_{\mathrm{agg}}(q)+B q^{-n}
$$

where $L_{\mathrm{X}}(q)=M_{\mathrm{X}} K_{\mathrm{X}}^{2} /\left[\left(q-q_{\mathrm{X}}\right)^{2}+K_{\mathrm{X}}^{2}\right]$, with $X=\mathrm{a}, \mathrm{b}$, and agg representing the side chain, backbone correlations and the inter-aggregate interference, respectively, and the $B q^{-n}(n=2.5-3.3)$ term represents the upturn at the lowest $q$. For each Lorentzian part, the peak position, $q_{\mathrm{X}}$, the half-width at half-maximum, $K$, and an intensity factor $M$ are the three fitting parameters, where the latter two parameters give the peak area $A_{\mathrm{X}}=\pi M K$. All fitting parameters of the Lorentzian functions are summarized in Table S1. The fitting curves are shown in Figure 3, which agree reasonably with the experimental results. $q_{\mathrm{a}}$ and $q_{\mathrm{b}}$ are very similar for all ionomer samples probably because they were synthesized from the same prepolymer and had a low ion content. $q_{\text {agg }}$, indicated with the arrow in Figure 3, reflects the interference between aggregates. The average distance $D_{\text {agg }}$ between those ionic aggregates can be evaluated from $q_{\text {agg }}$ as $D_{\text {agg }}=2 \pi / q_{\text {agg. }}$. For all ionomer samples, this $D_{\text {agg }}$ is plotted 
against $M_{\mathrm{n}}$ in the inset of Figure 3 .

Table S1. Structural parameters determined by fitting the X-ray scattering profiles at room temperature with Lorentzian functions.

\begin{tabular}{cccccccccc}
\hline samples & \multicolumn{3}{c}{$L_{\mathrm{a}}$} & \multicolumn{3}{c}{$L_{\mathrm{b}}$} & \multicolumn{3}{c}{$L_{\text {agg }}$} \\
& $M_{\mathrm{a}}$ & $K_{\mathrm{a}}$ & $q_{\mathrm{a}}$ & $M_{\mathrm{b}}$ & $K_{\mathrm{b}}$ & $q_{\mathrm{b}}$ & $M_{\text {agg }}$ & $K_{\text {agg }}$ & $q_{\text {agg }}$ \\
\hline $5 \mathrm{~K}-\mathrm{COONa}$ & 4420 & 2.2 & 13.4 & 3620 & 3.2 & 5.2 & 5590 & 0.6 & 0.65 \\
$9 \mathrm{~K}-\mathrm{COONa}$ & 4350 & 2.2 & 13.4 & 3600 & 2.5 & 5.2 & 5590 & 0.6 & 0.6 \\
$16 \mathrm{~K}-\mathrm{COONa}$ & 6700 & 2.5 & 13.4 & 5400 & 2.4 & 5.3 & 5300 & 0.5 & 0.45 \\
$6 \mathrm{~K}-\mathrm{SO}_{3} \mathrm{Na}$ & 3800 & 2.8 & 13.4 & 2960 & 2 & 5.4 & 9700 & 0.8 & 1.2 \\
$9 \mathrm{~K}-\mathrm{SO}_{3} \mathrm{Na}$ & 5400 & 2.8 & 13.4 & 3600 & 2.2 & 5.4 & 4600 & 0.9 & 1.1 \\
$16 \mathrm{~K}-\mathrm{SO}_{3} \mathrm{Na}$ & 5700 & 2.4 & 13.4 & 4683 & 2.2 & 5.4 & 4166 & 0.7 & 0.82 \\
\hline
\end{tabular}

\section{S5.2 Temperature-dependent SAXS scattering profiles}

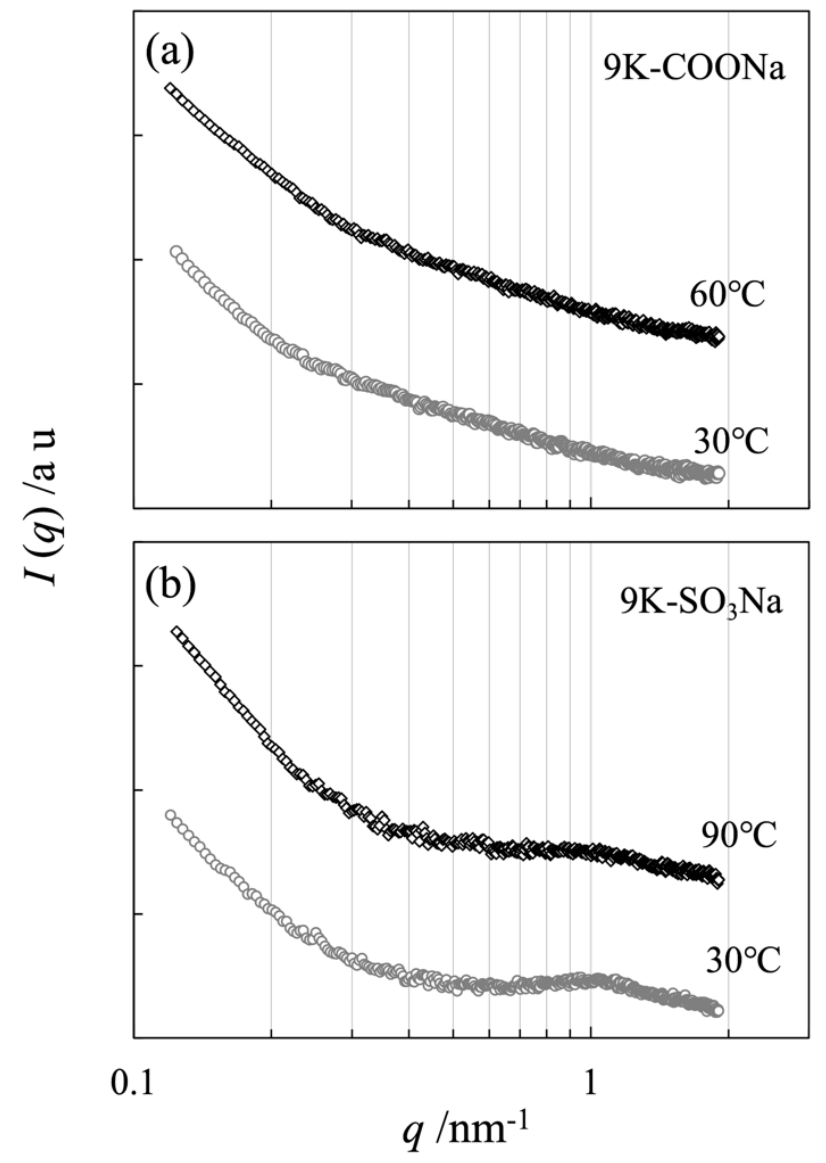

Figure S8. SAXS profiles $I(q)$ of $9 \mathrm{~K}-\mathrm{COONa}$ (a) and $9 \mathrm{~K}-\mathrm{SO}_{3} \mathrm{Na}(\mathrm{b})$ ionomers at temperatures as indicated. The data were first measured at $30^{\circ} \mathrm{C}$ (gray circle) and then at 60 or $90^{\circ} \mathrm{C}$ (black square) where the samples can rapidly flow. Scales for $I(q)$ are logarithmic with decades on the intensity scale shown by horizontal grid lines. The 
scattering peak of $M_{\mathrm{n}}-\mathrm{SO}_{3} \mathrm{Na}$ became a little broader at $90^{\circ} \mathrm{C}$, which may reflect slight suppression of aggregation of ionic groups (suppression of segregation from the polymeric matrix) on an increase of $T$ from $30^{\circ} \mathrm{C}$ to $90^{\circ} \mathrm{C}$.

\section{S5.3 Difference of associated network structures in $M_{n}-\mathrm{SO}_{3} \mathrm{Na}$ and $M_{\mathrm{n}}-\mathrm{COONa}$ ionomers}

In the $M_{\mathrm{n}}-\mathrm{SO}_{3} \mathrm{Na}$ and $M_{\mathrm{n}}$-COONa ionomers, the network of superbridges dynamically crosslinked by the salt group aggregates sustains the plateau of $G^{\prime}$ and the dissociation of those aggregates determines the terminal relaxation, as explained in the main text. Thus, it is informative to summarize how we can visualize that superbridge network. This summary is presented below. Because $E_{\mathrm{a}}$ for the dissociation $\left(25\right.$ and $39 \mathrm{~kJ} \mathrm{~mol}^{-1}$ for $M_{\mathrm{n}}-\mathrm{COONa}$ and $M_{\mathrm{n}}-\mathrm{SO}_{3} \mathrm{Na}$ at $20^{\circ} \mathrm{C}$; cf. section 3.2 in the main text) is much larger than $k_{\mathrm{B}} T$, the following summary naturally considers that the ion groups stay in the associated aggregates.

\section{$M_{\mathrm{n}}-\mathrm{SO}_{3} \mathrm{Na}$ system.}

A) In the $M_{\mathrm{n}}-\mathrm{SO}_{3} \mathrm{Na}$ ionomers, all aggregates of the salt groups appear to have a similar scattering power (as explained for Figure 8 in the main text) and thus a similar number $n_{\mathrm{S}}$ of salt groups per aggregate. For simplicity, we start with an assumption of no distribution of $n_{\mathrm{S}}$. Then, we subdivide the total number density of the $\mathrm{SO}_{3} \mathrm{Na}$ salt group, $2 v$ with $v$ being the chain number density, by this $n_{\mathrm{S}}$ to obtain the number density of the aggregates,

$$
v_{\text {agg }}=\frac{2 v}{n_{\mathrm{S}}}
$$

The following cartoon is for a case of $n_{\mathrm{S}}=4$.

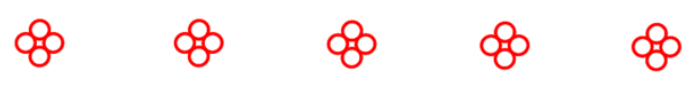

B) The number density of effective crosslinks, corresponding to the number density of the elastically active network strands (superbridges) $v_{\mathrm{X}}$, is estimated from the above $v_{\mathrm{agg}}$ as

$$
v_{\mathrm{x}}=\frac{f v_{\mathrm{agg}}}{2}
$$

where $f$ is the functionality of effective crosslink, i.e., the aggregate of the salt groups. The filled circles in the following cartoon indicate the aggregates serving as the effective end-crosslinks of the superbridge for a case of $f=4$.

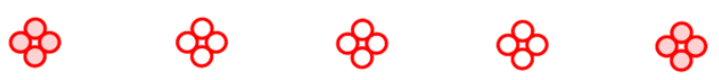

C) Each ionomer chain is supposed to connect two salt groups in the same or different aggregates to have a loop or connector conformation. (A dangling tail having a free salt group is considered later.) The effective crosslinks are located at the ends of a sequence of connectors. Thus, we can make such a sequence, identical to a skeleton of the superbridge, as illustrated below.

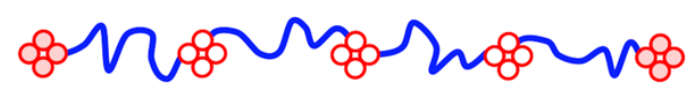


The remaining $n_{\mathrm{S}}-1$ salt groups in each effective crosslink (filled circles at the ends of the skeleton) connect the superbridge to the network, so that the connectors are added to those $n_{\mathrm{s}}-1$ salt groups as indicated below.

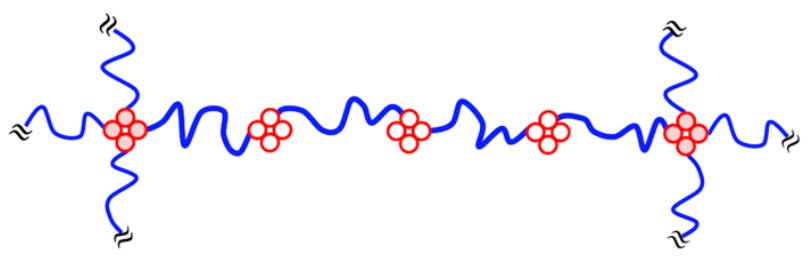

This network skeleton sustains the plateau of $G^{\prime}$.

D) The above cartoon still contains salt groups having no chain on them. Those groups would carry dangling loops and dangling tails (mostly the dangling loops because of the strong association of the $\mathrm{SO}_{3} \mathrm{Na}$ salt groups). Then, the network structure would be something shown below.

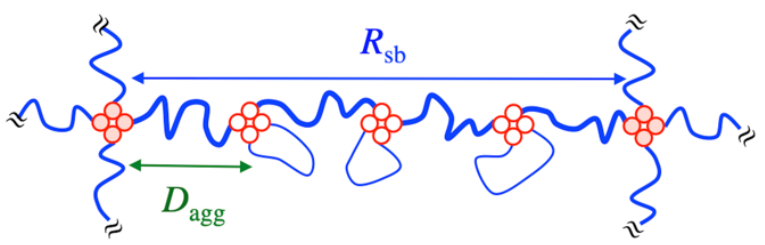

The end-to-end distance of the superbridge skeleton $R_{\mathrm{sb}}$ and the distance between the aggregates $D_{\mathrm{agg}}$ do not agree with each other $\left(R_{\mathrm{sb}}>D_{\mathrm{agg}}\right)$, and the plateau of $G^{\prime}$ reflects $R_{\mathrm{sb}}$.

E) The above argument is oversimplified because it does not consider a larger dangling loop originated from several dangling tails. The following cartoon would be closer to reality.

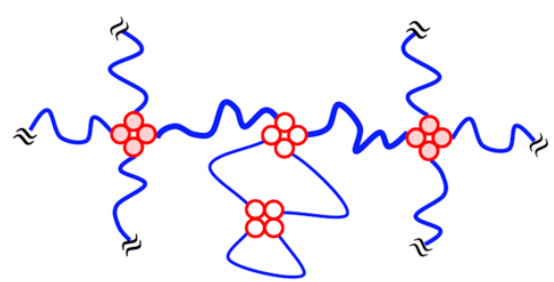

In this cartoon, the salt domain in the larger loop is drawn to have the same aggregation number as those in the superbridge backbone, to be consistent with the experimental observation for the X-ray scattering profile and the plateau modulus summarized in Figure 8 of the main text.

F) The above arguments A-E are still over-simplified because no distribution is considered for the number of ionomer chains per superbridge skeleton, $g^{\circ}$. The real network structure in $M_{\mathrm{n}}-\mathrm{SO}_{3} \mathrm{Na}$ would include longer and shorter skeletons illustrated below, with an average of $R_{\mathrm{sb}}$ for all skeletons coinciding with the averaged end-to-end distance of the network strand reflected in the plateau modulus.
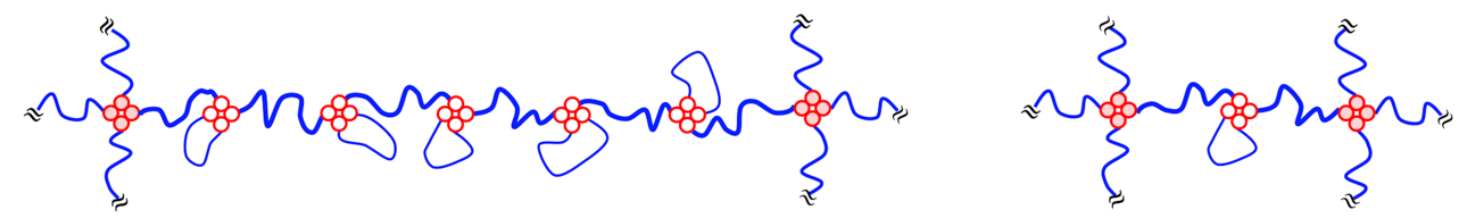
This $R_{\mathrm{sb}}$ distribution (not detectable with SAXS for $M_{\mathrm{n}}-\mathrm{SO}_{3} \mathrm{Na}$ ) may have a direct consequence in the nonlinear rheological behavior at high Wi. A shorter skeleton increases its tension at a smaller strain compared to a longer skeleton (because the full-stretch limit is smaller for the shorter skeleton) and triggers the macroscopic rupture.

\section{Mn-COONa system}

As judged from the similarity of $g_{\mathrm{G}}$ (similarity of $G_{\mathrm{pl}}$ ) between the $M_{\mathrm{n}}-\mathrm{COONa}$ and $M_{\mathrm{n}}-\mathrm{SO}_{3} \mathrm{Na}$ ionomers (cf. Figure 8c in the main text), we may assume that the basic network skeleton is more or less similar in these ionomers. At the same time, the $M_{\mathrm{n}}$-COONa ionomers should have a considerable distribution of the number of salt groups per aggregate $n_{\mathrm{S}}$, and $D_{\text {agg }}$ measures the distance between two large(est) aggregates. Thus, as a trial of mimicking the network structure in the $M_{\mathrm{n}}-\mathrm{COONa}$ ionomer, we may start with the carton shown in the above argument $\mathbf{D}$ and transfer a pair of the salt groups carrying the dangling loops inside the superbridge skeleton to the other aggregates as

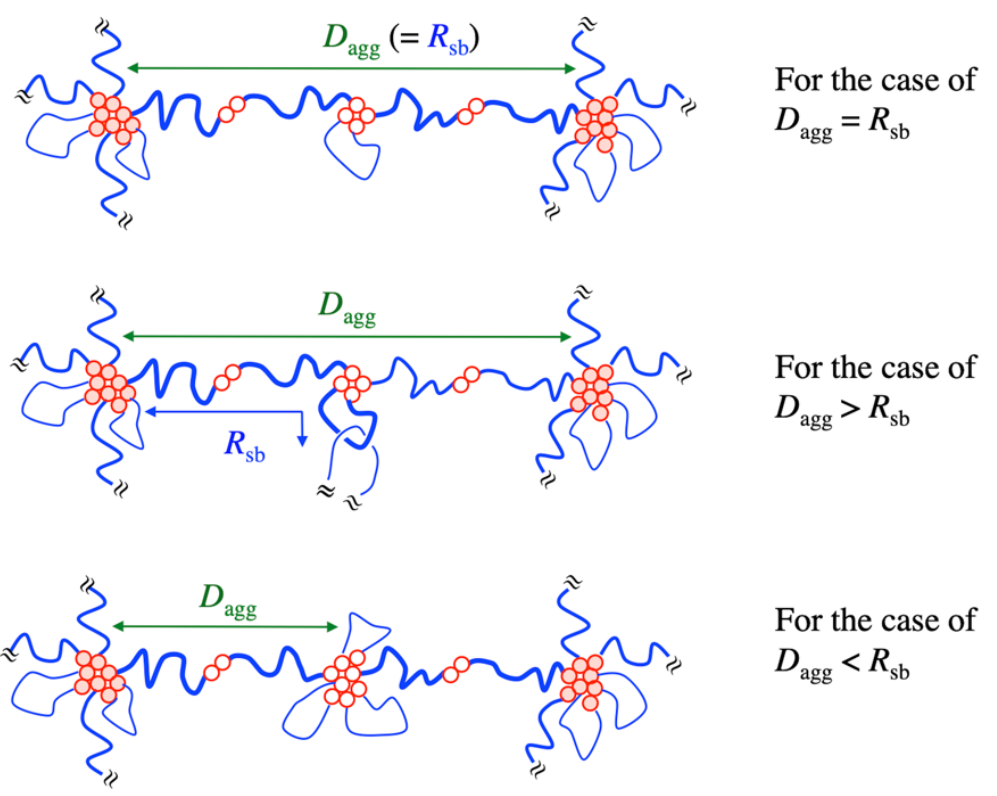

In the top cartoon, the end aggregates (effective crosslinks for the superbridge skeleton) contain more salt groups compared to the inner aggregates. For this case, X-ray scattering detects the distance between the end aggregates, namely, $D_{\mathrm{agg}}=R_{\mathrm{sb}}$.

In the middle cartoon, a knotted loop serves as the effective crosslink despite the aggregate sustaining this loop does not contain so many salt groups. For this case, $D_{\mathrm{agg}}$ can be larger than $R_{\mathrm{sb}}$.

In the bottom cartoon, some of the aggregates inside the superbridge skeleton are as large as the end aggregates. Those large inner aggregates have two connectors and many dangling loops (and tails). For this case, $D_{\mathrm{agg}}<R_{\mathrm{sb}}$.

As judged from the behavior of $M_{\mathrm{n}}$-COONa ionomers explained in the main text, a decrease of the 
number $g_{\mathrm{S}}$ of chains between large (X-ray detected) aggregates with increasing $M_{n}$ (cf. red squares in Figure $8 \mathrm{c}$ ), the network structure in those ionomers seems to shift from the top cartoon to the middle and further to the bottom cartoon on an increase of $M_{n}$. The mechanism of this change is not clear, and a future study is desired.

\section{S6 Nonlinear Rheological data of ionomers with $M_{n}=5 \mathrm{~K}$ and $16 \mathrm{~K}$}

\section{S6.1 Startup Shear Rheology}

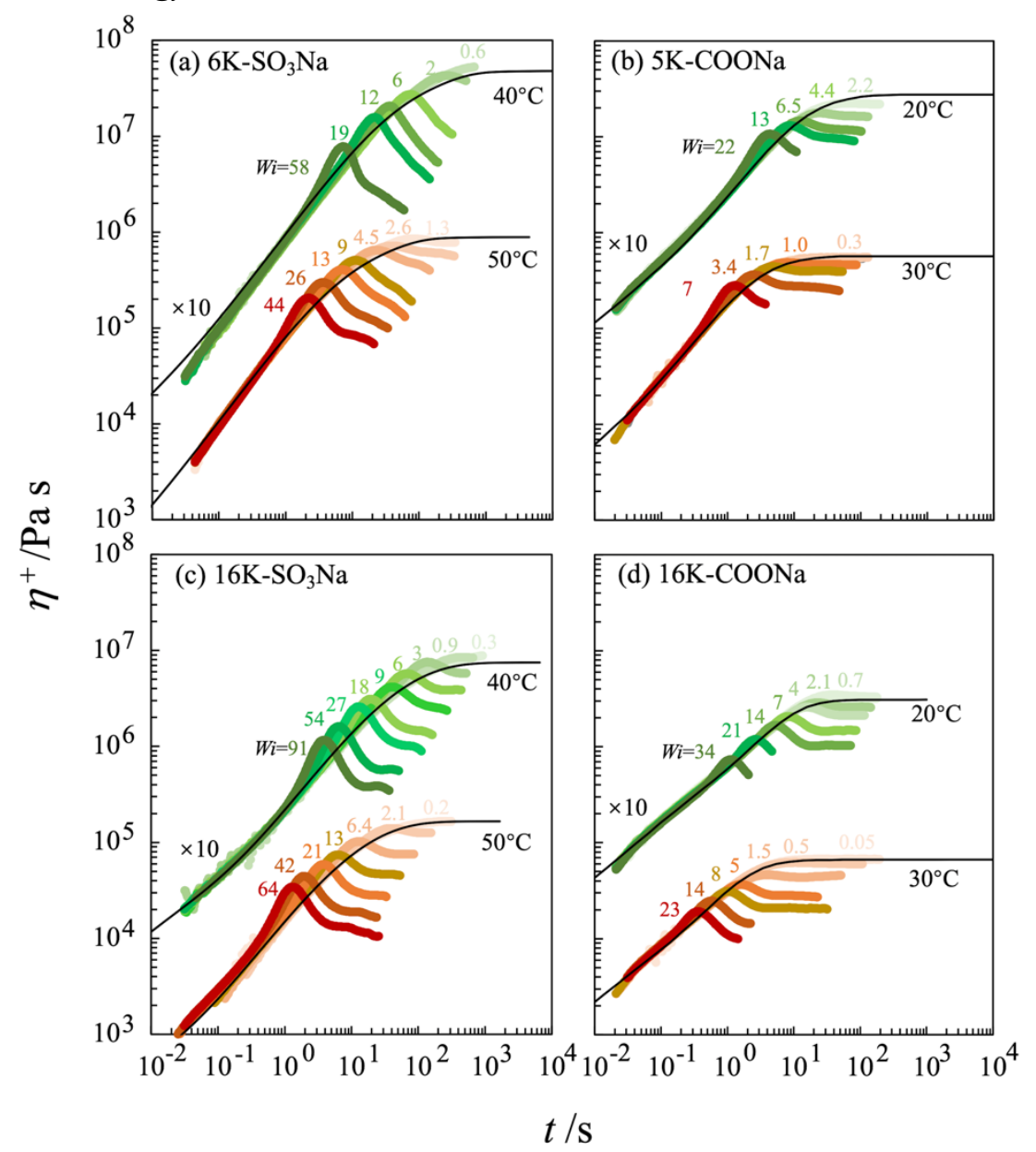

Figure S9. Plots of shear stress growth coefficient $\eta^{+}$against time $t$ at various $W i$ for (a) $6 \mathrm{~K}-\mathrm{SO}_{3} \mathrm{Na}$, (b) $5 \mathrm{~K}-\mathrm{COONa}$, (c) $16 \mathrm{~K}-\mathrm{SO}_{3} \mathrm{Na}$ and (d) $16 \mathrm{~K}-\mathrm{COONa}$, respectively. In each panel, the data at lower $T$ are shifted upward by a factor of 10 (shown as " $\times 10 "$ ) to avoid heavy overlap of the plots.

Figure $\mathrm{S} 9$ shows that the growth of $\eta^{+}$during short $t$ for $M_{\mathrm{n}}-\mathrm{SO}_{3} \mathrm{Na}$ and $M_{\mathrm{n}}-\mathrm{COONa}$ ionomers with $M_{\mathrm{n}}=5 \mathrm{~K}$ and $16 \mathrm{~K}$ follows the LVE curve independent of $W i$, indicating the linearity of any material under sufficiently small strain. However, for $W i>1$, we note that $\eta^{+}$of the ionomers at long $t$ shows a transient hardening above the LVE curve along with the overshoot before reaching the steady viscosity $\eta$. For very large $W i$, the melt rupture (edge fracture) behavior appears around the otherwise steady viscosity region, leading to the stress drops after overshoot, which is related to the strong strain-induced dissociation that causes the flow instability. Similar results are also observed for 
ionomers with $M_{\mathrm{n}}=9 \mathrm{~K}$ in Figure 9.

Figure S10 summarizes the plots of shear stress $\sigma\left(=\dot{\gamma} \eta^{+}\right)$against the strain $\gamma(=\dot{\gamma} t)$ for $M_{\mathrm{n}}-\mathrm{SO}_{3} \mathrm{Na}$ and $M_{\mathrm{n}}-\mathrm{COONa}$ ionomers with $M_{\mathrm{n}}=5 \mathrm{~K}$ and $16 \mathrm{~K}$. We find that $\gamma_{\text {peak }}$ (unfilled blue circles) is quite insensitive to $W i$ and increases with increasing $M_{\mathrm{n}}$. The former feature suggests that the stress exhibits the overshoot when the flow deforms the transient network strands to a given extent specified by $\gamma_{\text {peak }}$ and begins to disrupt the network, and the later feature reflects the larger extensibility for larger telechelic chains before strain-induced dissociations.

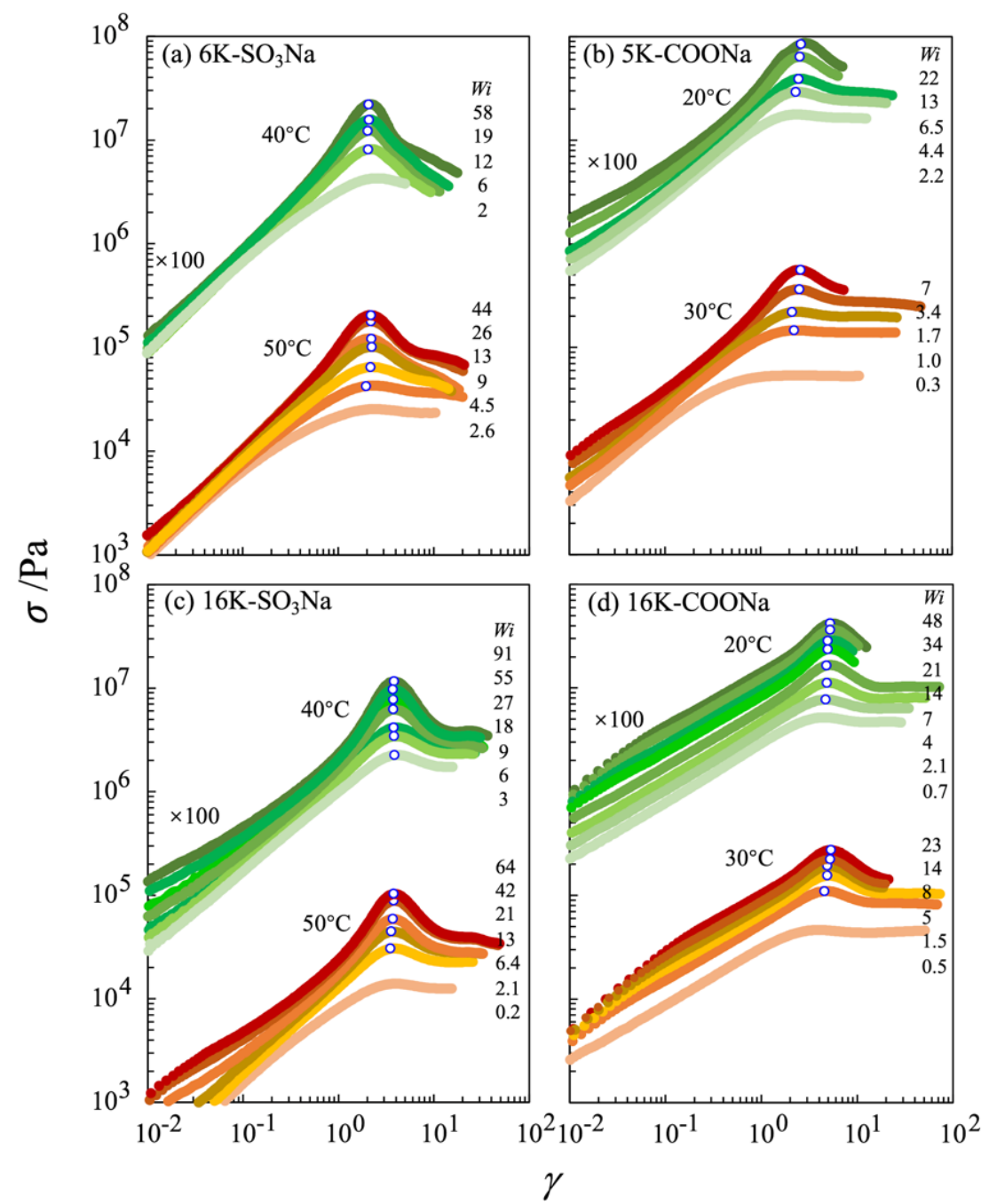

Figure $\mathrm{S} 10$. Plots of shear stress $\sigma$ against strain $\gamma$ at various $W i$ for (a) $6 \mathrm{~K}-\mathrm{SO}_{3} \mathrm{Na}$, (b) $5 \mathrm{~K}-\mathrm{COONa},(\mathrm{c}) 16 \mathrm{~K}-\mathrm{SO}_{3} \mathrm{Na}$ and (d) $16 \mathrm{~K}-\mathrm{COONa}$, respectively. In each panel, the data at lower $T$ are shifted upward by a factor of 100 (shown as " $\times 100 "$ ) to avoid heavy overlap of the plots. The blue circles show the stress peak. 


\section{S6.2 Startup Elongation Rheology}

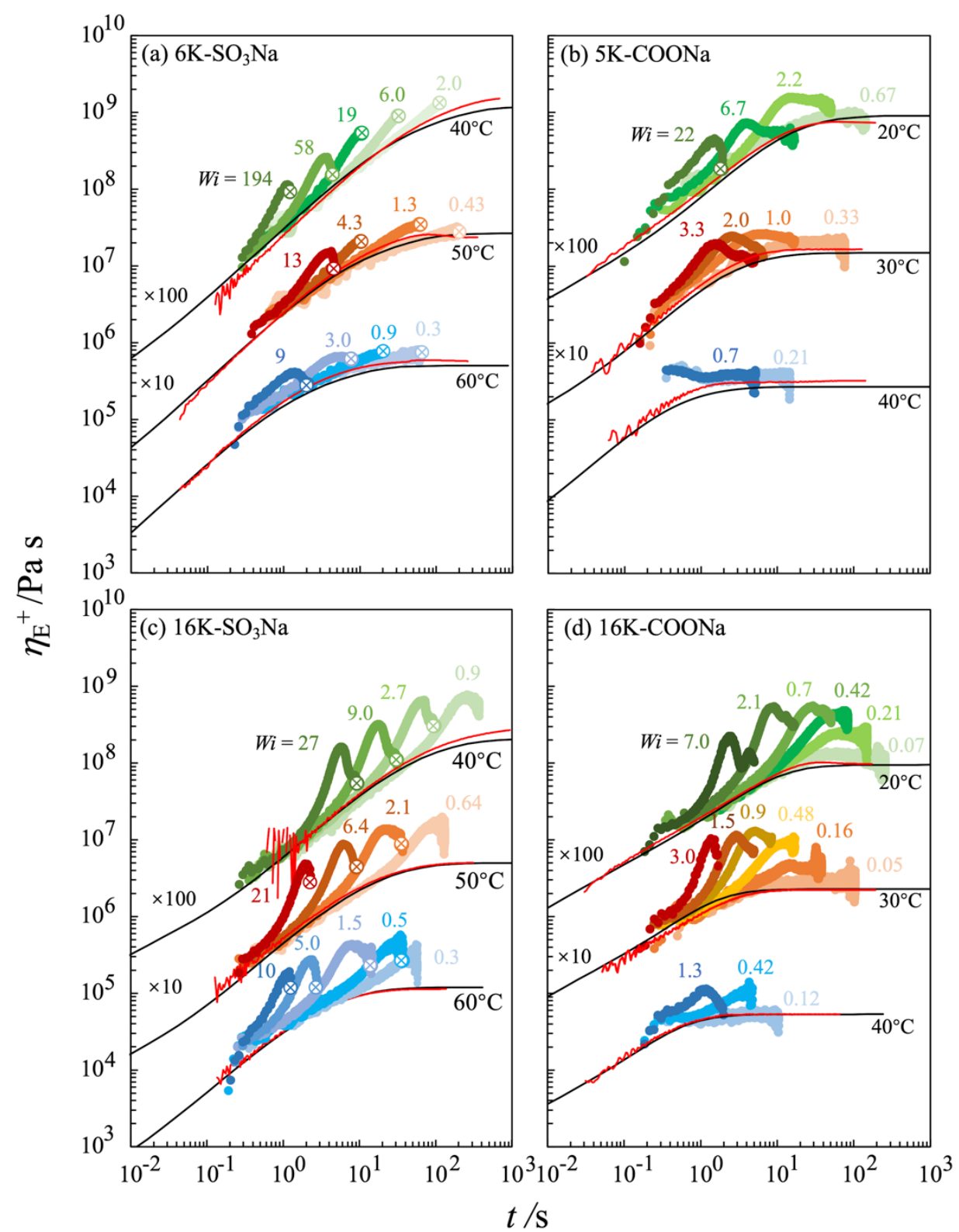

Figure S11. Plots of elongational stress growth coefficient $\eta_{\mathrm{E}}^{+}$against time $t$ for (a) $6 \mathrm{~K}-\mathrm{SO}_{3} \mathrm{Na}$, (b) $5 \mathrm{~K}-\mathrm{COONa}$, (c) $16 \mathrm{~K}-$ $\mathrm{SO}_{3} \mathrm{Na}$, and (d) $16 \mathrm{~K}-\mathrm{COONa}$. In each panel, the data at different $T$ are shifted vertically by factors as indicated (following the symbol " $\times$ "). The crossmarked circle $(\otimes)$ indicates that the sample ruptured at that point.

Figure $\mathrm{S} 11$ shows the elongational stress growth coefficient $\eta_{\mathrm{E}}^{+}(t, W i)$ measured for $M_{\mathrm{n}}-\mathrm{SO}_{3} \mathrm{Na}$ and $M_{\mathrm{n}}$-COONa ionomers with $M_{\mathrm{n}}=5 \mathrm{~K}$ and $16 \mathrm{~K}$ under constant rate $\left(\dot{\varepsilon}_{\mathrm{H}}\right)$ elongation at several $T$. For all ionomers, we found that $\eta_{\mathrm{E}}{ }^{+}$is close to the LVE envelope (curves) at short $t$, as naturally expected under small strains at short $t$. However, $\eta_{\mathrm{E}}{ }^{+}$increases above that envelope at intermediate $t$, exhibits strong strain hardening behavior followed by a peak, and then approaches the steady flow state. The $M_{\mathrm{n}}-\mathrm{SO}_{3} \mathrm{Na}$ ionomers with different $M_{\mathrm{n}}$ show a macroscopic fracture behavior at a point shown with the crossmarked circle $(\otimes)$. In contrast, only $M_{\mathrm{n}}-\mathrm{COONa}$ ionomer with $5 \mathrm{~K}$ exhibits melt fracture at 
$W i=22$. Thus, $M_{\mathrm{n}}-\mathrm{COONa}$ is more ductile compared to $M_{\mathrm{n}}-\mathrm{SO}_{3} \mathrm{Na}$ (as also noted under shear; cf. Figures S9 and S10).

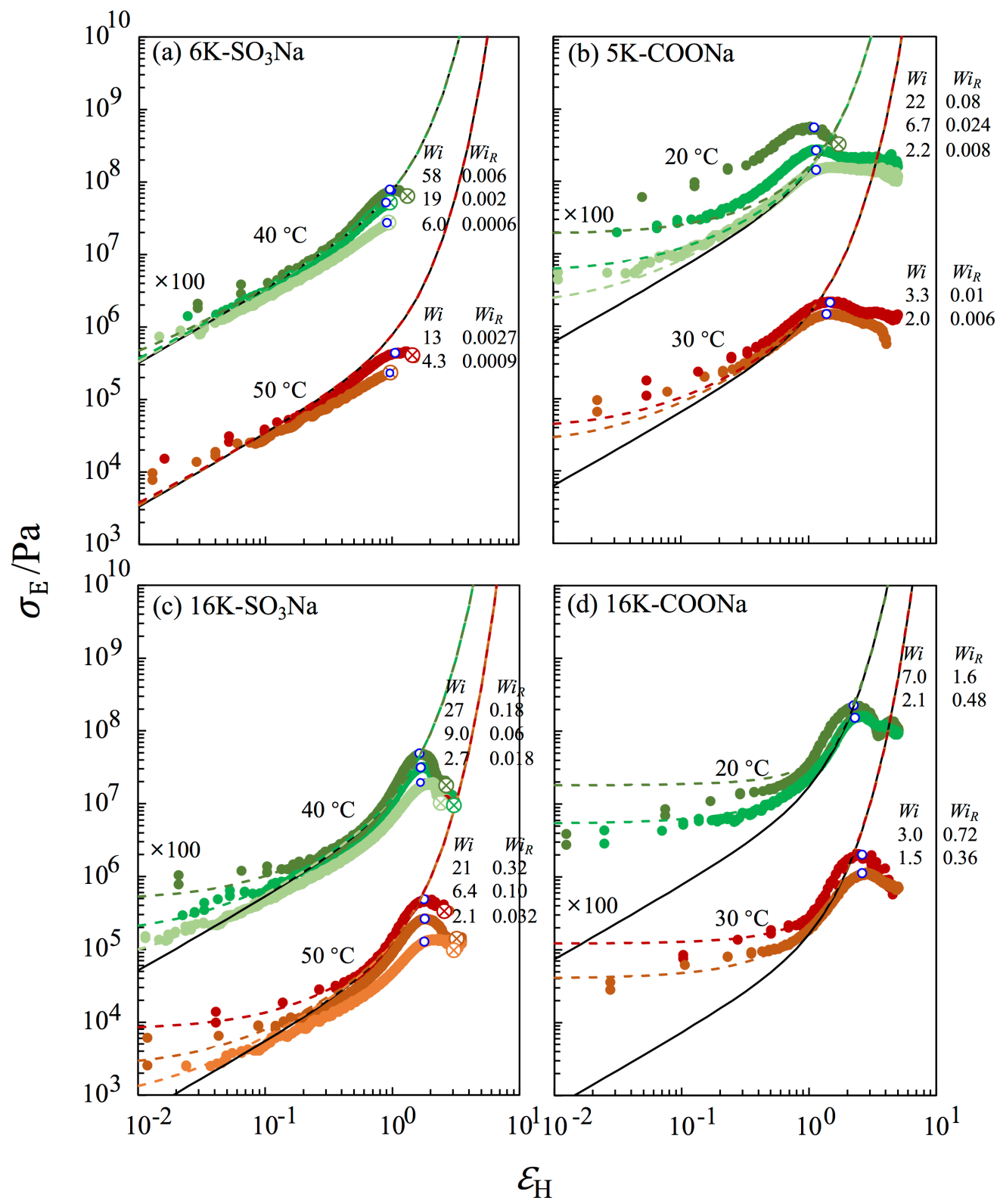

Figure S12. Plots of tensile stress $\sigma_{\mathrm{E}}$ against the Hencky strain $\varepsilon_{\mathrm{H}}$ for (a) $6 \mathrm{~K}-\mathrm{SO}_{3} \mathrm{Na}$, (b) $5 \mathrm{~K}-\mathrm{COONa}$, (c) $16 \mathrm{~K}-\mathrm{SO}_{3} \mathrm{Na}$ and (d) 16K-COONa. In each panel, the data at different $T$ are shifted vertically by factors as indicated (following the symbol " $\times$ "). The cross-marked circle $(\otimes)$ indicates that the sample ruptured at that point. The unfilled blue circles show the stress peak. The solid and dashed curves (black and colored curves), respectively, show the neo-Hookean stress calculated as $\sigma_{\mathrm{E}, \text { neo-H }}=G_{\mathrm{pl}}\left(\lambda^{2}-1 / \lambda\right)$ and $\sigma_{\mathrm{E}, \text { neo-H }}=G_{\mathrm{pl}}\left(\lambda^{2}-1 / \lambda\right)+3 \eta_{\mathrm{R}} \dot{\varepsilon}_{\mathrm{H}}$, i.e., without and with a contribution from the fast and local Rouse relaxation.

Figure $\mathrm{S} 12$ summarizes the plots of tensile stress $\sigma_{\mathrm{E}}$ against the Hencky strain $\varepsilon_{\mathrm{H}}$ for $M_{\mathrm{n}}-\mathrm{SO}_{3} \mathrm{Na}$ and $M_{\mathrm{n}}-\mathrm{COONa}$ ionomers with $M_{\mathrm{n}}=5 \mathrm{~K}$ and $16 \mathrm{~K}$. We found that $\varepsilon_{\mathrm{H} \text {,peak }}$ (unfilled blue circles) is quite insensitive to $W i$ and increases with increasing $M_{\mathrm{n}}$, which would result from a mechanism similar to that under shear. Namely, the stress overshoot occurs when the flow deforms the transient network 
strands to a given extent ( $\varepsilon_{\mathrm{H} \text {,peak }}$; see unfilled blue circles) corresponding to the onset of network disruption/yielding. $\varepsilon_{\mathrm{H}, \text { peak }}$ increases with increasing $M_{\mathrm{n}}$, which indicates a larger extensibility before the stress over shoot peak for larger $M_{\mathrm{n}}$ (as similar to the situation under shear).

\section{S7 Invariants of stress and strain tensors}

An anonymous reviewer suggested that some detail of yielding could be resolved with the aid of invariants of the stress and strain tensors. These invariants are examined below.

The deviatoric part (traceless part) of the stress tensor, $\sigma_{\mathrm{d}}$, includes no isotropic pressure term and thus straightforwardly represents the mechanical state of a material under deformation. For our ionomers modelled as a neo-Hookean rubber before the yielding, this stress tensor can be expressed as $\sigma_{\mathrm{d}}=G_{\mathrm{p} 1} \Gamma_{\mathrm{d}}$, where $G_{\mathrm{pl}}$ is the experimentally observed plateau modulus and $\Gamma_{\mathrm{d}}=\Gamma-\{\operatorname{tr}(\Gamma) / 3\} \mathbf{I}$ is a deviatoric part of the Finger strain tensor $\Gamma$. This $\sigma_{d}$ is rewritten in terms the shear strain $\gamma$ or the stretch ratio $\lambda$ as $^{3}$

$$
\begin{gathered}
\text { under shear: } \sigma_{\mathrm{d}}=G_{\mathrm{pl}}\left(\begin{array}{ccc}
2 \gamma^{2} / 3 & \gamma & 0 \\
\gamma & -\gamma^{2} / 3 & 0 \\
0 & 0 & -\gamma^{2} / 3
\end{array}\right) \\
\text { under elongation: } \sigma_{\mathrm{d}}=G_{\mathrm{pl}}\left\{\lambda^{2}-\frac{1}{\lambda}\right\}\left(\begin{array}{ccc}
2 / 3 & 0 & 0 \\
0 & -1 / 3 & 0 \\
0 & 0 & -1 / 3
\end{array}\right)
\end{gathered}
$$

The first invariant of $\sigma_{\mathrm{d}}, J_{1} \equiv \operatorname{tr}\left(\sigma_{\mathrm{d}}\right)$, is zero by definition. The second and third invariants, $J_{2}$ and $J_{3}$, are given by ${ }^{3}$

$$
\begin{aligned}
& J_{2} \equiv-\frac{1}{2}\left[\operatorname{tr}\left(\sigma_{d}^{2}\right)-\left\{\operatorname{tr}\left(\sigma_{d}\right)\right\}^{2}\right]=\left\{\begin{array}{l}
-\frac{1}{3} G_{\mathrm{pl}}{ }^{2} \gamma^{2}\left(\gamma^{2}+3\right) \text { under shear } \\
-\frac{1}{3} G_{\mathrm{pl}}^{2}\left(\lambda^{2}-\frac{1}{\lambda}\right)^{2} \text { under elongation }
\end{array}\right. \\
& J_{3} \equiv \operatorname{det}\left(\sigma_{d}\right)=\left\{\begin{array}{c}
\frac{1}{27} G_{\mathrm{pl}}^{3} \gamma^{4}\left\{2 \gamma^{2}+9\right\} \text { under shear } \\
\frac{2}{27} G_{\mathrm{pl}}^{3}\left\{\lambda^{2}-\frac{1}{\lambda}\right\}^{3} \text { under elongation }
\end{array}\right.
\end{aligned}
$$


$J_{2}^{[\text {peak }]}$ at the stress overshoot peak is equivalent to the von Mises stress: $J_{2}^{[\text {peak }]}=-\left\{\sigma_{\mathrm{M}}^{[\text {peak }]}\right\}^{2} / 3 \quad(\mathrm{cf}$. eq S6 and eqs 4 and 5 in the main text). For the $M_{\mathrm{n}}-\mathrm{SO}_{3} \mathrm{Na}$ ionomers, Figure $14 \mathrm{a}$ in the main test has shown a close agreement of $\sigma_{\mathrm{M}}^{\text {[peak] }}$ under shear and elongation and thus the agreement of $J_{2}^{\text {[peak] }}$ under these flow conditions. This result suggests that no significant flow-induced disruption of the ionomer network occurs in $M_{\mathrm{n}}-\mathrm{SO}_{3} \mathrm{Na}$ up to the stress overshoot peak. In contrast, for the $M_{\mathrm{n}}$ COONa ionomers, Figure $14 \mathrm{~b}$ demonstrated considerable deviation of $\sigma_{\mathrm{M}}^{\text {[peak] }}$ (and thus of $J_{2}^{\text {[peak] }}$ ) under shear and elongation, which suggests that the flow-induced disruption significantly occurs for the network therein but is followed by its rapid reformation, as discussed in the main text.

Now, we examine $J_{3}^{\text {[peak] }}$ of our ionomers evaluated from $\gamma_{\text {peak }}$ and $\lambda_{\mathrm{H} \text {,peak }}=\exp \left(\varepsilon_{\mathrm{H} \text {,peak }}\right)$ shown in Figure 13 in the main text. Plots of those $J_{3}^{[\text {peak] }}$ against $M_{\mathrm{n}}$ are shown below in Figure S13. For the $M_{\mathrm{n}}$-COONa ionomers (Figure $\mathrm{S} 13 \mathrm{~b}$ ), a significant difference is noted for $J_{3}^{\text {[peak] }}$, s under shear and elongation, possibly due to the flow-induced disruption followed by rapid reformation explained above.

For the $M_{\mathrm{n}}-\mathrm{SO}_{3} \mathrm{Na}$ ionomers (Figure S13a), $J_{3}^{[\text {peak] }}$, s under shear and elongation exhibit similar changes with $M_{\mathrm{n}}$ but a considerable difference in the magnitude (by a factor of $\sim 3$ ) is also noted. This result, combined with the agreement of $\sigma_{\mathrm{M}}^{[\text {peak }]}\left(=\sqrt{-3 J_{2}^{[\text {peak }]}}\right)$ and/or $\Delta \sigma^{\text {[peak }]}$ under shear and elongation (Figure 14a in the main text), suggests that the yielding of the $M_{\mathrm{n}}-\mathrm{SO}_{3} \mathrm{Na}$ ionomers is specified by a limited aspect of the stress $\left(\sigma_{\mathrm{M}}^{[\text {peak }]}\right.$ and/or $\Delta \sigma^{[\text {peak }]}$ both being related to the shear stress component), not by full aspects of stress represented by $J_{i}^{[\text {peak }]}$ with $i=1-3$. This feature of the yielding in the $M_{\mathrm{n}}-\mathrm{SO}_{3} \mathrm{Na}$ ionomers deserves further attention.
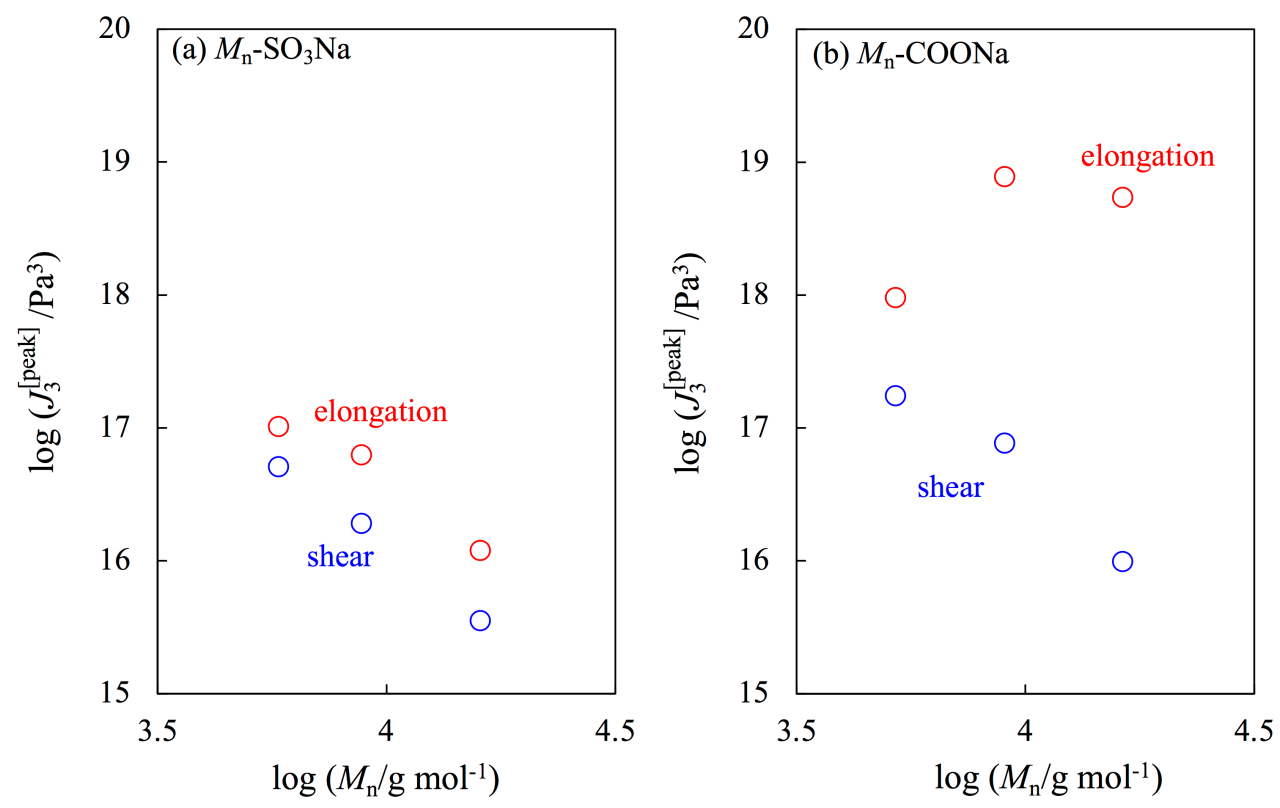

Figure S13: Double logarithmic plots of third invariant of deviatoric stress tensor, $J_{3}^{\text {[peak] }}$, against $M_{\mathrm{n}}$ for (a) $M_{\mathrm{n}}-\mathrm{SO}_{3} \mathrm{Na}$ and (b) $M_{\mathrm{n}}-\mathrm{COONa}$. 
Here, we turn our attention to the strain and examine how it determines the yielding in our ionomers. For this purpose, we focus on the Finger strain tensor ${ }^{3} \Gamma$ shown below.

$$
\begin{gathered}
\text { for shear: } \Gamma=\left(\begin{array}{ccc}
1+\gamma^{2} & \gamma & 0 \\
\gamma & 0 & 0 \\
0 & 0 & 0
\end{array}\right) \\
\text { for elongation: } \Gamma=\left(\begin{array}{ccc}
\lambda^{2} & 0 & 0 \\
0 & \lambda^{-1} & 0 \\
0 & 0 & \lambda^{-1}
\end{array}\right)
\end{gathered}
$$

Here, we have assumed an incompressible condition that automatically gives the third invariant $I_{3}=$ $\operatorname{det}(\Gamma)=1$. The first and second invariants of $\Gamma, I_{1}$ and $I_{2}$, are specified as ${ }^{3}$

$$
\begin{aligned}
& I_{1} \equiv \operatorname{tr}(\Gamma)=\left\{\begin{array}{l}
\gamma^{2}+3 \text { for shear } \\
\lambda^{2}+\frac{2}{\lambda} \text { for elongation }
\end{array}\right. \\
& I_{2} \equiv-\frac{1}{2}\left[\operatorname{tr}\left(\Gamma^{2}\right)-\{\operatorname{tr}(\Gamma)\}^{2}\right]=\left\{\begin{array}{l}
\gamma^{2}+3 \text { for shear } \\
2 \lambda+\frac{1}{\lambda^{2}} \text { for elongation }
\end{array}\right.
\end{aligned}
$$

It should be noted that $I_{1}^{[\text {peak }]}$ and $I_{2}^{\text {[peak }]}$ for shear exactly coincide with each other.

From $\gamma_{\text {peak }}$ and $\lambda_{\mathrm{H} \text {,peak }}=\exp \left(\varepsilon_{\mathrm{H}, \text { peak }}\right)$ shown in Figure 13 in the main text, we evaluated $I_{1}^{[\text {peak }]}$ and $I_{2}^{[\text {peak }]}$ at the stress overshoot peak. In Figure S14, these invariants are plotted against $M_{\mathrm{n}}$.

If the ionomer network is not flow-disrupted up to the stress overshoot peak and the yielding occurs at a given strain, each of the strain invariants at the stress peak, each of $I_{j}^{[\text {peak }}(j=1-3)$ is expected to be the same under the shear and elongation. For $M_{\mathrm{n}}-\mathrm{COONa}$ examined in Figure $\mathrm{S} 14 \mathrm{~b}, I_{2}^{[\text {peak] }}$ 's under the shear and elongation are close to each other (cf. small filled circles) but a significant difference is noted for $I_{1}^{[\text {peak] }}$ 's (cf. large unfilled circes). This result, combined with those discussed for Figures $\mathrm{S} 13 \mathrm{~b}$ and Figure $14 \mathrm{~b}$ in the main text, suggests that the yielding behavior of $M_{\mathrm{n}}-\mathrm{COONa}$ is fully determined by neither the strain nor the stress (possibly because of the flow-induced disruption and rapid reformation of the ionomer network occurring prior to the stress overshoot peak). 

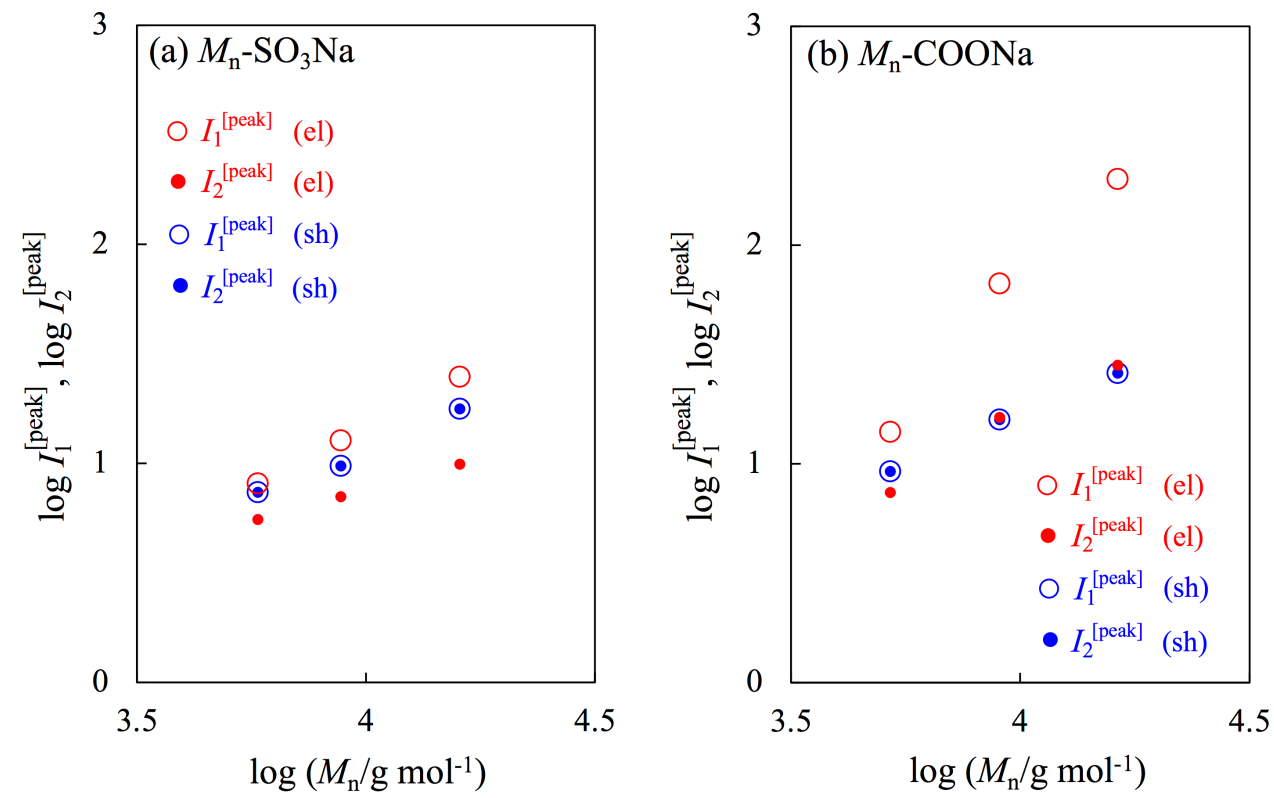

Figure S14: Double logarithmic plots of first and second invariants of strain tensor, $I_{1}^{[\text {peak }]}$ and $I_{2}^{\text {[peak }]}$, against $M_{\mathrm{n}}$ for (a) $M_{\mathrm{n}}-\mathrm{SO}_{3} \mathrm{Na}$ and (b) $M_{\mathrm{n}}-\mathrm{COONa}$.

In contrast, for $M_{\mathrm{n}}-\mathrm{SO}_{3} \mathrm{Na}$, the first invariant $I_{1}^{[\text {peak] }}$ follows the above expectation; $I_{1}^{\text {[peak] }}$ 's under shear and elongation are in close agreement (within $30 \%$ ) irrespecive of $M_{\mathrm{n}}$; see large unfilled circles in Figure S14a. A similar magnitude of agreement is noted also for $I_{2}^{\text {[peak] }}$ 's under shear and elongation, although a difference between these $I_{2}^{\text {[peak] }}$ 's seems to be gradually enhanced on an increase of $M_{\mathrm{n}}$ (cf. small filled circles). Thus, for each $M_{\mathrm{n}}-\mathrm{SO}_{3} \mathrm{Na}$ ionomer, the strain charcaterized by $I_{j}^{\text {[peak] }}$ with $j=1-3$ appears to be (nearly) the same at the stress overshoot peak under shear and elongation. Nevertheless, this result does not mean that the mechanical state characterized by the stress tensor $\sigma_{\mathrm{d}}=G_{\mathrm{pl}}[\Gamma-\{\operatorname{tr}(\boldsymbol{\Gamma}) / 3\} \mathbf{I}]$ at that peak is the same under shear and elongation, as already noted in Figure S13. Namely, the yielding does not reflect full aspects of $\sigma_{\mathrm{d}}$ (characterized by all of $J_{i}^{\text {[peak }]}$ with $i=1-3$ ). Instead, a particular component of $\sigma_{\mathrm{d}}$ (limited aspect of $\sigma_{\mathrm{d}}$ ), the shear stress component giving the elastic energy considered in the von Mises criterion and specifying the maximum shear stress considered in the Tresca criterion, seems to be the factor governing the yielding of $M_{\mathrm{n}}-\mathrm{SO}_{3} \mathrm{Na}$. Unfortunately, at this moment, we cannot further specify if the yielding is governed just by the elastic energy or the maximum shear stress (or both). This problem deserves further attention.

\section{S8 Viscoelastic Behavior of Acidic Prepolymers}

For the acidic prepolymers $M_{\mathrm{n}}-\mathrm{COOH}$ and $M_{\mathrm{n}}-\mathrm{SO}_{3} \mathrm{H}$, the terminal viscoelastic relaxation time $\tau$ $\left(=\left[G^{\prime}(\omega) / \omega G^{\prime \prime}(\omega)\right]_{\omega \rightarrow 0}\right)$ increases and the corresponding relaxation intensity $G_{1}\left(\sim\left[G^{\prime} \omega\right]_{\omega=1 / \tau}\right)$ decreases with increasing $M_{\mathrm{n}}$, as can be noted from Figures 4 and 5 in the main text (see unfilled 
symbols). This behavior is similar to that of unentangled Rouse chains $\left(\tau_{1} \sim M^{2}\right.$ and $\left.G_{1} \sim M^{-1}\right)$. Thus, we examined if the acidic prepolymers exhibit the Rouse dynamics. The results are shown in Figure $\mathrm{S} 15$, where normalized storage and loss moduli of the prepolymers, $G^{\prime} M_{\mathrm{n}} / M_{\mathrm{r}}$ and $G^{\prime \prime} M_{\mathrm{n}} / M_{\mathrm{r}}$, are plotted against a reduced frequency, $\omega M_{\mathrm{n}}^{2} / M_{\mathrm{r}}^{2}$, where $M_{\mathrm{r}}$ is chosen to be the lowest $M_{\mathrm{n}}$ in the series of the $M_{\mathrm{n}}-\mathrm{COOH}$ prepolymers (in panel a) or the $M_{\mathrm{n}}-\mathrm{SO}_{3} \mathrm{H}$ prepolymers (in panel $\mathrm{b}$ ). The solid curves show the prediction of the Rouse model (in the absence of molecular weight distribution),

$$
\frac{M}{M_{\mathrm{r}}} G^{*}(\omega)=\frac{\rho R T}{M_{\mathrm{r}}} \sum_{q=1}^{N} \frac{i \omega \tau_{q}}{1+i \omega \tau_{q}}
$$

with

$$
\tau_{q}=\tau \frac{\sin ^{2}\left(\frac{\pi}{2(N+1)}\right)}{\sin ^{2}\left(\frac{q \pi}{2(N+1)}\right)} \quad(q=1,2, . ., N)
$$

Here, $\rho\left(=1.07 \mathrm{~g} / \mathrm{cm}^{3}\right)$ is the mass density, ${ }^{4} R$ is the gas constant, and $\tau_{q}$ is the characteristic time of the $q$-th mode of the Rouse chain composed of $N$ springs $\left(N+1\right.$ beads); $N=M_{\mathrm{n}} / M_{\mathrm{K}}$ with $M_{\mathrm{K}}(=770)^{5}$ being the Kuhn segment molecular weight of PiBA. The data of all prepolymers agree reasonably with each other and are close to the Rouse prediction, as noted in Figure S15. This result confirms that the prepolymers behave as ordinary unentangled polymers, possibly because of the lack of significant hydrogen bonding in those polar prepolymers (as already discussed for Figures 4 and 5 in the main text). The disagreement between the $G^{\prime \prime}$ data at high $\omega$ and the Rouse calculation is attributable to a contribution of the glassy relaxation not incorporated in eq S12. 


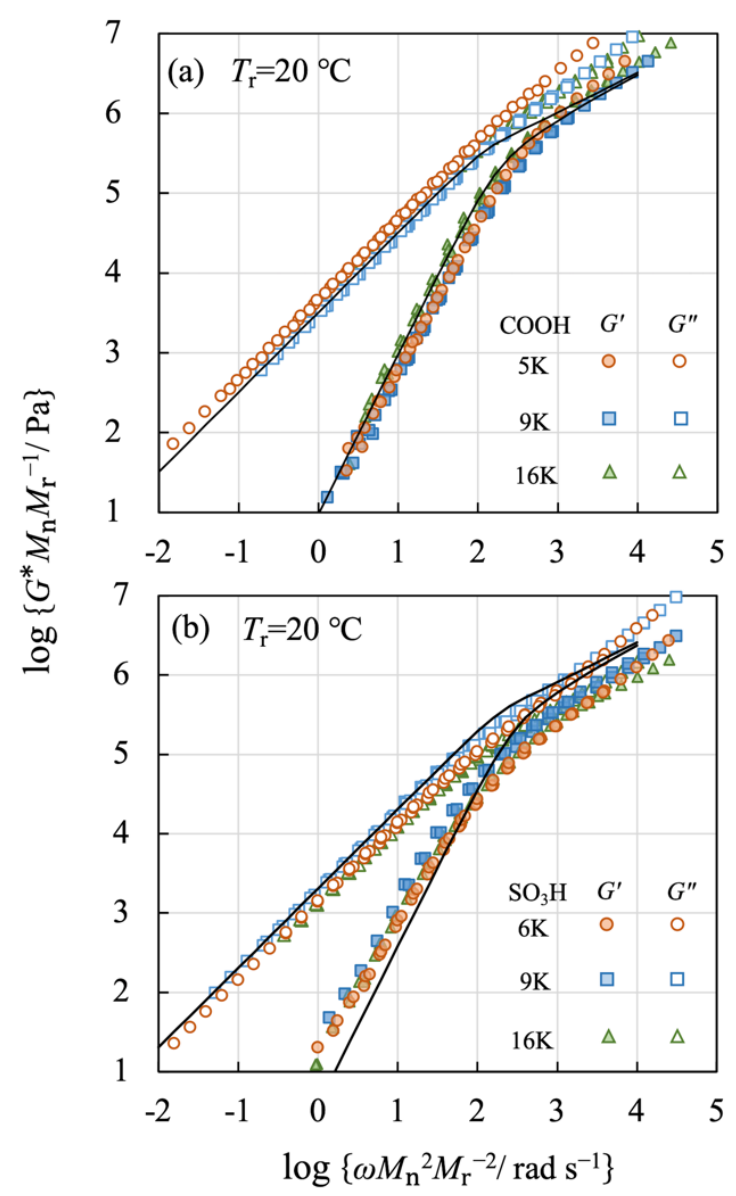

Figure S15: Plots of reduced complex modulus $G^{*} M_{\mathrm{n}} / M_{\mathrm{r}}$ against a reduced frequency $\omega M_{\mathrm{n}}{ }^{2} / M_{\mathrm{r}}{ }^{2}$ for (a) $M_{\mathrm{n}}-\mathrm{COOH}$ and (b) $M_{\mathrm{n}}-\mathrm{SO}_{3} \mathrm{H}$ acidic prepolymers. $M_{\mathrm{r}}$ is the lowest $M_{\mathrm{n}}$ in the series of the $M_{\mathrm{n}}-\mathrm{COOH}$ prepolymers (in panel (a) or the $M_{\mathrm{n}}-$ $\mathrm{SO}_{3} \mathrm{H}$ prepolymers (in panel (b)).

\section{References:}

(1) Lai, J. T.; Filla, D.; Shea, R. Functional polymers from novel carboxyl-terminated trithiocarbonates as highly efficient RAFT agents. Macromolecules 2002, 35 (18), 6754-6756.

(2) Liu, Y. Synthesis and Characterization of Well-defined, Amphiphilic, Ionic Copolymers. University of Akron, 2011.

(3) Dill, E. H. Continuum Mechanics. Elasticity, Plasticity, Viscoelasticity. CRC Press: Boca Raton, 2006.

(4) Yamaguchi, T.; Wang, B. G.; Matsuda, E.; Suzuki, S.; Nakao, S. I. Prediction and estimation of solvent diffusivities in polyacrylate and polymethacrylates. Journal of Polymer Science Part B: Polymer Physics 2003, 41 (12), 1393-1400.

(5) Zhang, Z.; Liu, C.; Cao, X.; Wang, J.-H. H.; Chen, Q.; Colby, R. H. Morphological Evolution of Ionomer/plasticizer Mixtures during a Transition from Ionomer to Polyelectrolyte. Macromolecules 2017, 50 (3), 963-971. 